Special issue of the 2nd International Conference on Computational and Experimental Science and Engineering (ICCESEN 2015)

\title{
A Bias Reducing Approach for Some Robust Estimators by Predicting Roughness in Case of Kernel Estimation
}

\author{
N. GÜNDÜZ*, C. AYdin AND E. BAŞAR \\ Gazi University, Faculty of Science, Statistics Department, Ankara, Turkey
}

\begin{abstract}
In the density estimation it is known that estimators are heavily biased. We applied a bias reducing approach to improve some quantile estimators for Weibull distribution having different parameter values and contamination level. In this study, we estimate the bias for any quantile value and obtained biased reduced smoothed distribution function by simulation study for random samples of size 40 . Then, the mean square error of some robust quantile estimators and variances are obtained from biased reduced smoothed distribution function. Furthermore, we obtained sampling distribution of roughness and sampling distribution of estimated bias related some quantile estimators
\end{abstract}

DOI: 10.12693/APhysPolA.130.422

PACS/topics: $02.70 . \mathrm{Rr}, 02.50 .-\mathrm{r}$

\section{Introduction}

In this study, some quantile-based robust estimators are investigated for the Weibull distribution. When data is contaminated, the estimators are affected strongly in the negative manner. So, it is important to use robust estimators as a measure of distribution properties for analyzing data in the case of contamination with outliers. The first proposals about kernel smoothing for distribution functions estimates has been made by Nadaraya [1] and Azzalini [2]. After that, smoothing empirical distribution function by kernel estimation and obtaining estimators based on smoothed distribution is frequently applied.

Generally, for small data sets and in the case of outlier, Fernholz [3] has proven that the mean square error (MSE) of the estimators obtained from the smoothed distribution function is less than the MSE of estimators obtained from the empirical distribution function. Hubert et al. [4], stated the results of a simulation study conducted for the kernel smoothing to random samples that are taken from a gamma distribution with various parameters including the cases of contamination. It is declared that a great reduction is achieved in the MSE of smoothed quantile-based estimators depending on a simulation study applied for Weibull distribution with different parameter value [5].

Furthermore, when kernel estimation is obtained in order to achieve smoothness, it is known that the estimators are heavily biased. For that reason, it is necessary to improve the estimators by using a bias reducing approach. In this study, we applied a bias reduction approach to some quantile estimator for Weibull distribution having different parameter values and contamination level.

For this purpose, first we obtained kernel smoothed distribution function, than we estimate the bias for any

\footnotetext{
*corresponding author; e-mail: ngunduz@gazi.edu.tr
}

quantile value, after that we obtained biased reduced smoothed distribution function by simulation study for random samples of size 40 . And then the MSE of robust estimators and variances are obtained from biased reduced smoothed distribution function.

As a quantile-based robust statistics, the median (med), interquartile range (IQR), quartile skewness (QS) and octile skewness (OS) are considered. In the case of contamination with different proportion, the behavior of mentioned statistics is investigated by a simulation study and results are summarized.

In the second section of the study we introduce basic concepts and definitions such as quantile function and some robust quantile estimators. Kernel smoothing and estimation of the roughness and bandwidth selection which are crucial parameters in kernel smoothing are provided in Section 3. Simulation study takes place in the Section 4. Results of simulation study are summarized and tabulated in Section 5. In Section 6 theory is applied to lifetimes of transplanted kidneys data. Finally, conclusion stated in the last section.

\section{Basic concepts}

Let $\left\{x_{1}, x_{2}, \ldots, x_{n}\right\}$ be an independent and identically distributed random sample, with sample size $n$, drawn from an absolutely continuous distribution function $F(x)$ with probability density function $f(x)$. The population quantile function is defined as the first place that the value of distribution function is at least $p$ and is given as follows:

$$
Q(p)=\inf \{x: p \leq F(x)\}, \quad 0 \leq p \leq 1 .
$$

Empirical distribution function is

$$
\begin{gathered}
F_{n}(x)=\hat{F}(x)=\frac{1}{n} \sum_{i=1}^{n} I_{(-\infty, x]}\left(x_{i}\right), \\
I_{(-\infty, x]}(u)= \begin{cases}1, & u \in(-\infty, x], u \leq x \\
0, & u \notin(-\infty, x], u>x\end{cases}
\end{gathered}
$$


Accordingly, empirical quantile function is

$$
Q_{n}(p)=\inf \left\{x: p \leq F_{n}(x)\right\}, \quad 0 \leq p \leq 1 .
$$

As it is seen from the definition, the empirical quantile function (estimator of quantile function) is inverse of the empirical distribution function. In this study, some robust quantile based estimators such as, median as a location estimator, interquartile range as a scale estimator, quartile skewness and octile skewness as a measure of skewness are investigated for Weibull distribution $[4,6,7]$.

\section{Kernel smoothing}

An empirical distribution function estimates the distribution function of a random variable by assigning equal probability to each observation in a sample. It is discontinuous at many points. Kernel smoothing is applied to achieve a smoother empirical distribution function so we have a continuous estimate of distribution function, which makes possible to estimate the density of a random variable based on an observed sample. Kernel-based estimator of distribution function is given as follows [1]

$$
\tilde{F}_{n, h}(x)=\frac{1}{n} \sum_{i=1}^{n} K\left(\frac{x-X_{i}}{h}\right),
$$

where $K(t)$ is distribution function, having a density $k(t)$ that is symmetric around zero and a bandwidth $h$ that controls the degree of smoothness. Since the choice of kernel function $K(t)$ is less important than the choice of bandwidth, we took under consideration only Epanechnikov kernel [8, 9]. Smoothed distribution function, $\tilde{F}_{n, h}(x)$, is continuous, so for any $p, 0 \leq p \leq 1$, it is possible to obtain a smoothed quantile estimate. In practice, the range of the smoothed distribution function is equally partitioned to many sections; each section matches a quantile value, so we have a smoothed quantile estimate for each section in one-to-one correspondence. We use interpolation to obtain other quantile estimates. If a random variable $X$ has a distribution function $F(x)$, that is differentiable twice and has continuous second derivative, while $n \rightarrow \infty, h \rightarrow 0, n h \rightarrow \infty$, the expected value and the variance of the smoothed distribution function estimator $\tilde{F}_{n, h}(x)$ is given as follows

$$
\begin{aligned}
& E\left(\tilde{F}_{n, h}(x)\right)=F(x)+\frac{1}{2} h^{2} f^{\prime}(x) \mu_{2}(k)+o\left(h^{2}\right), \\
& V\left(\tilde{F}_{n, h}(x)\right)=\frac{F(x)(1-F(x))}{n} \\
& \quad-\frac{2 h f(x) c}{n}+o\left(\frac{h}{n}\right),
\end{aligned}
$$

where $\mu_{2}(k)=\int_{-\infty}^{\infty} t^{2} k(t) \mathrm{d} t$ and $c=\int_{-\infty}^{\infty} t k(t) K(t) \mathrm{d} t$. For the Epanechnikov kernel we have $\mu_{2}(k)=1$, the constant $c$ is $c=0.2875$ [2].

As it is seen in Eq. (5), smoothed distribution function estimator is biased. We look for a bias reducing approach. The MSE of smoothed distribution function,

$$
\operatorname{MSE}\left(\tilde{F}_{n, h}(x)\right)=E\left[\tilde{F}_{n, h}(x)-F_{X}(x)\right]^{2} .
$$

Since we are interested in all quantile based estimator, it is best to minimize IMSE:

$$
\begin{aligned}
& \operatorname{IMSE}(h)=\operatorname{IMSE}\left(\tilde{F}_{n, h}(x)\right)= \\
& \quad \int_{-\infty}^{\infty} \operatorname{Var}\left(\tilde{F}_{n, h}(x)\right) \mathrm{d} x+\int_{-\infty}^{\infty}\left[\operatorname{Bias}\left(\tilde{F}_{n, h}(x)\right)\right]^{2} \mathrm{~d} x, \\
& \left(\operatorname{Bias}\left(\tilde{F}_{n, h}(x)\right)=E\left(\tilde{F}_{n, h}(x)\right)-F_{X}(x)=\right. \\
& \left.\frac{1}{2} h^{2} f^{\prime}(x) \mu_{2}(k)+o\left(h^{2}\right)\right) .
\end{aligned}
$$

By applying elementary integral operations

$$
\begin{aligned}
& \int_{-\infty}^{\infty} \operatorname{Var}\left(\tilde{F}_{n, h}(x)\right) \mathrm{d} x= \\
& \int_{-\infty}^{\infty}\left[\frac{F(x)(1-F(x))}{n}\right] \mathrm{d} x+\int_{-\infty}^{\infty}\left[-\frac{2 h f(x) c}{n}\right] \mathrm{d} x= \\
& \int_{-\infty}^{\infty}\left[\frac{F(x)(1-F(x))}{n}\right] \mathrm{d} x-\frac{2 h c}{n}+o\left(h^{2}\right), \\
& \int_{-\infty}^{\infty}\left[\operatorname{Bias}\left(\tilde{F}_{n, h}(x)\right)\right]^{2} \mathrm{~d} x= \\
& \int_{-\infty}^{\infty}\left[\frac{1}{2} h^{2} f^{\prime}(x) \mu_{2}(k)\right]^{2} \mathrm{~d} x+o\left(\frac{h}{n}\right)= \\
& \underbrace{\int_{-\infty}^{\infty} h^{4} \mu_{2}^{2}(k)}_{=\text {Roughness }=R} f^{\prime}(x))^{2} d x=\frac{1}{4} h^{4} \mu_{2}^{2}(k) R .
\end{aligned}
$$

We obtain the IMSE as follows

$$
\begin{aligned}
& \operatorname{IMSE}(h)=\operatorname{IMSE}\left(\tilde{F}_{n, h}(h)\right)= \\
& \quad \int_{-\infty}^{\infty}\left[\frac{F(x)(1-F(x))}{n}\right] \mathrm{d} x-\frac{2 h c}{n}+\frac{1}{4} h^{4} \mu_{2}^{2}(k) R .
\end{aligned}
$$

IMSE expression is a function of bandwidth. We are looking for the minimum value of IMSE

$$
\begin{gathered}
\frac{\partial \operatorname{IMSE}(h)}{\partial h}=0-\frac{2 c}{n}+h^{3} \mu_{2}^{2}(k) R=0 \\
\Rightarrow h=\left(\frac{2 c}{n \mu_{2}^{2}(k) R}\right)^{-\frac{1}{3}} .
\end{gathered}
$$

We expect that this bandwidth would minimize the IMSE. In this sense, it is optimum. We see that, optimal bandwidth value depends on roughness $R$, so it must be estimated.

\section{Bias reducing in kernel estimation}

By ordering the random sample we obtained order statistics, than we found sample variance and inter quartile range (IQR). We made first bandwidth prediction as follows [10]

$$
h_{\text {first }}=2.34 \min \left(\hat{\sigma}_{n}, \frac{\mathrm{IQR}_{n}}{1.349}\right) n^{-\frac{1}{5}} .
$$

In the case of outliers, we know that sample variance is strongly effected while IQR statistics is less affected. Since breakdown point of IQR is 0.25 . If there is an outlier, the IQR statistics will define the first bandwidth 
otherwise sample variance will define it. Consequently, first bandwidth selection will be close to optimum and robust in any case. We use the predicted first bandwidth value to predict roughness of the random sample. We use the predicted bandwidth value to obtain the estimator of the second derivative of density function by kernel estimation

$$
\tilde{f}_{n, h}^{\prime \prime}(x)=\frac{1}{n h_{\text {first }}^{3}} \sum_{i=1}^{n} k^{\prime \prime}\left(\frac{x-X_{i}}{h_{\text {first }}}\right) .
$$

We have the estimated roughness value for all points in the range (related to this random sample). In order to obtain $\left(\tilde{f}_{n, h}^{\prime \prime}(x)\right)$ roughness estimates by kernel estimation we need to define a working interval, which can be based on the range of the random sample. We expand the working interval by adding 2 times bandwidth length to both sides

Upper working interval $=x_{(\min )}+2 \times h_{\text {first }}$,

Lower working interval $=x_{(\max )}-2 \times h_{\text {first }}$.

Since, for every point of the working interval, we want to obtain kernel estimation we define step size and step number to control the sensitivity of the results

\section{Working interval length $=$ step size $\times$ step number.}

When step size decreases, step number increases and vice versa. So it is possible to establish a control. We construct a coordinate grid on working interval by step size and step number. So we estimate $\tilde{f}_{n, h}^{\prime \prime}(x)$ values for many points, according to step size and step number value. We defined the points of the working interval in which kernel estimation is applied for every in the following manner

$$
\begin{aligned}
& x=\text { working interval lower bound }+ \text { step size } \times k, \\
& k=1,2,3, \ldots, \\
& \tilde{f}_{n, h}^{\prime \prime}(x)=\frac{1}{n h^{3}}\left[k^{\prime \prime}\left(\frac{x-X_{1}}{h_{\text {first }}}\right)+k^{\prime \prime}\left(\frac{x-X_{2}}{h_{\text {first }}}\right)+\ldots\right. \\
& \left.\quad+k^{\prime \prime}\left(\frac{x-X_{n}}{h_{\text {first }}}\right)\right], \\
& \tilde{f}_{n, h}^{\prime \prime}(x+\text { stepsize })=\frac{1}{n h^{3}}\left[k^{\prime \prime}\left(\frac{x+\text { stepsize }-X_{1}}{h_{\text {first }}}\right)\right. \\
& +k^{\prime \prime}\left(\frac{x+\text { stepsize }-X_{2}}{h_{\text {first }}}\right)+\ldots \\
& \left.+k^{\prime \prime}\left(\frac{x+\text { stepsize }-X_{n}}{h_{\text {first }}}\right)\right] .
\end{aligned}
$$

We need roughness estimate for that random sample, so we get as follows

$$
\hat{R}\left(X_{1}, X_{2}, \ldots, X_{n}\right)=-\frac{1}{n} \sum_{i=1}^{n} \tilde{f}_{n, h}^{\prime \prime}\left(X_{i}\right) .
$$

We obtained roughness estimate as sample mean from the smoothed estimator of second derivative of density function. Afterword, according to minimizing IMSE approach the optimal bandwidth selection would be

$$
h_{\mathrm{opt}}=\left(\frac{2 c}{\hat{R}}\right)^{\frac{1}{3}} n^{-\frac{1}{3}} .
$$

This is an estimate of bandwidth based on that random sample and it is optimum. Now we can obtain the smoothed distribution function estimate by applying kernel estimation

$$
\tilde{F}_{n, h_{\mathrm{opt}}}(x)=\frac{1}{n} \sum_{i=1}^{n} K\left(\frac{x-X_{i}}{h_{\mathrm{opt}}}\right) .
$$

As kernel estimation is applied for producing $\tilde{f}_{n, h}^{\prime \prime}(x)$ estimates, same things are repeated to achieve $\tilde{F}_{n, h_{\text {opt }}}(x)$ estimates. However, smoothed distribution function estimator is biased. To apply a bias reducing approach we have to estimate bias, and so we need the smoothed estimator of the first derivative of density function.

$$
\begin{aligned}
& \operatorname{Bias}\left(\tilde{F}_{n, h}(x)\right)=E\left(\tilde{F}_{n, h}(x)\right)-F_{X}(x)= \\
& \frac{1}{2} h^{2} f^{\prime}(x) \mu_{2}(k)+o\left(h^{2}\right) .
\end{aligned}
$$

Finally we obtain bias reduced smoothed distribution function estimator

$$
\tilde{\tilde{F}}_{n, h}(x)=\tilde{F}_{n, h}(x)-\frac{1}{2} h_{\text {opt }}^{2} \tilde{f}^{\prime}(x) \mu_{2}(k) .
$$

When we subtract the estimated bias from the smoothed distribution function, we obtain a new function which does not satisfy distribution function property. By using interpolation we deduce the distribution function property again.

\section{Simulation results}

In order to illustrate the reduction in variance and MSE of the different quantile-based estimators, we performed a simulation study on different Weibull distributions. In particular we considered random sample of size $n=40$ from Weibull distributions with scale parameter $\beta=1$ and shape parameter $\alpha=1.5,5$. Note that increasing the shape parameter makes the distribution more symmetric. We also considered contaminated samples. Data sets with "right" contamination is generated from a $N(3,(1 / 25))$ and all simulations are repeated 1000 times.

Firstly, sample mean of estimated roughness and bandwidth and their variances are tabulated in Table I. Additionally, we also present their sampling distributions in Fig. 1a and b. Secondly, mean of estimated bias and variance for different estimators in the case of different contamination level for Weibull $(1.5,1)$ are tabulated in Table II. Further we also illustrate sampling distributions of estimated bias in Fig. 1c and d. Thirdly in Table III, the ratio of variances of estimates bias of quantile estimators and median are presented. Finally we tabulated results from bias reduced smoothed distribution function for considered quantile-based estimators (Table IV).

For these Weibull distributions, we obtained quite different results about roughness and bandwidth. Actually, 
they are corresponding to each other. For Weibull(1.5, 1), we know that it is a flat distribution so we hope that roughness will be small, as it actually is. As a result we are allowed to use wider bandwidth. In the case of $\operatorname{Weibull}(5,1)$ we know that density is located in a small interval, because of that it has a sharp slope which is the reason of large roughness. So, it causes to use very small bandwidth in order to be sensitive. Our simulation results support this idea, which supplies better kernel smoothing especially in the case of contamination. Roughness and bandwidth are inverse to each other, they act in opposite directions. When one increases the other decreases.

TABLE I

Sampling distributions of estimated roughness and estimated bandwidth.

\begin{tabular}{c|c|c|c|c|c}
\hline \hline & Contamination & $\begin{array}{c}\text { Sample mean of } \\
\text { estimated roughness }\end{array}$ & $\begin{array}{c}\text { Sample variance of } \\
\text { estimated roughness }\end{array}$ & $\begin{array}{c}\text { Sample mean of } \\
\text { estimated bandwidth }\end{array}$ & $\begin{array}{c}\text { Sample var. of } \\
\text { estimated banwidth }\end{array}$ \\
\hline Weibull & no & 4.311779 & 5.679187 & 0.238634 & 0.001414 \\
$(1.5,1)$ & $5 \%$ & 3.035389 & 3.765998 & 0.273892 & 0.002673 \\
& $10 \%$ & 2.165819 & 2.844849 & 0.312298 & 0.00405 \\
\hline Weibull & no & 89.9205 & 2031.63 & 0.085825 & 0.000142 \\
$(5,1)$ & $5 \%$ & 67.0354 & 1497.13 & 0.096488 & 0.000287 \\
& $10 \%$ & 52.1652 & 1124.24 & 0.106141 & 0.00044
\end{tabular}

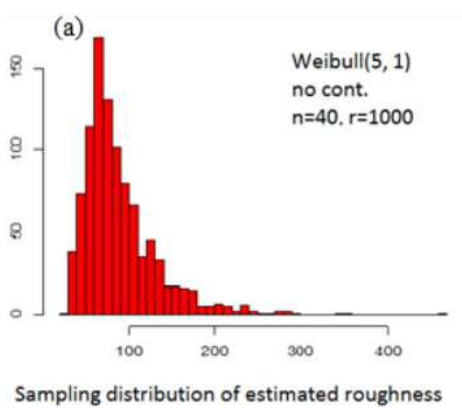

(d)

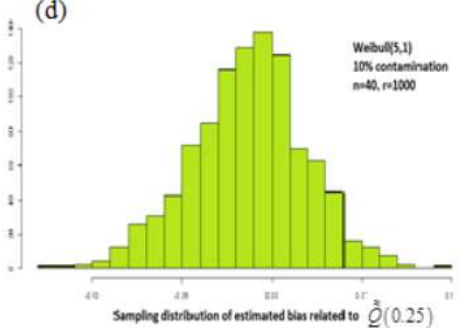

(b)

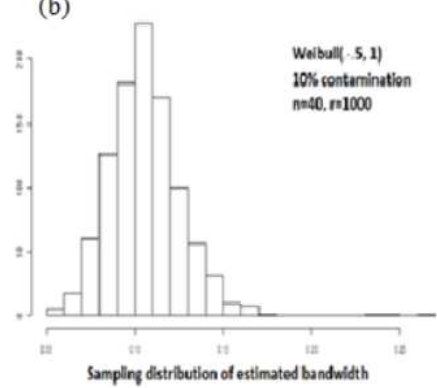

(e)

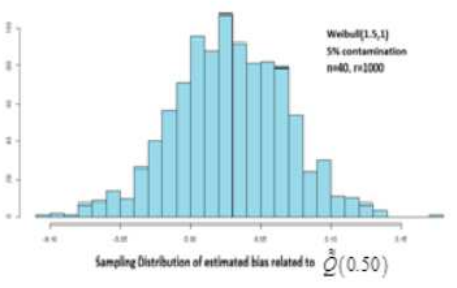

(c)

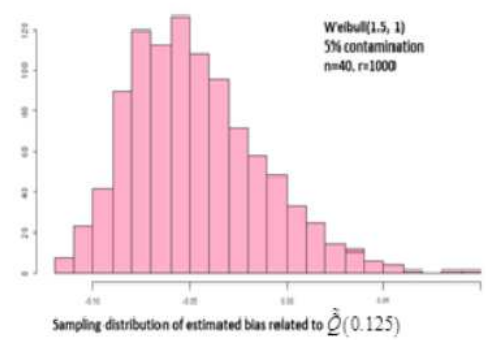

(f)

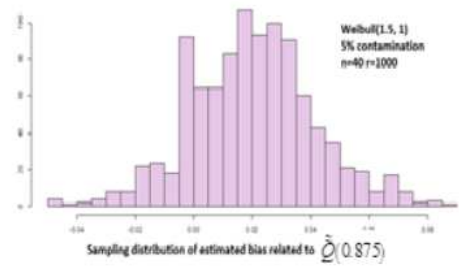

Fig. 1. Some sampling distribution functions.

The simulation results show that for both $\operatorname{Weibull}(1.5,1)$ and $\operatorname{Weibull}(5,1)$ the variance of estimated bias of considered estimators does not change with respect to contamination level.

In Table III, for $\operatorname{Weibull}(1.5,1)$ we see that the variance of the left side quantile estimators is the same with the variance of the median estimator, while the variance of the right side quantile estimators decreases sharply (except for the first cell). For $\operatorname{Weibull}(5,1)$ we see that the variance of estimated bias sharply decreases for small and large order of quantile estimators, while moving from median in both direction (except for the first cell).
The variance of estimated bias for median is same in both cases of Weibull distribution.

From Table IV, with the exception of the variance of $\tilde{\tilde{Q}}_{0.125}$, the variance of the other biased reduced smoothed estimators decreases as it is expected for $\operatorname{Weibull}(1.5,1)$. We see that for $\operatorname{Weibull}(5,1)$ for all quantile estimators the variance decreases, although small, in some cases.

\section{Real data example}

This bias reducing approach is applied to a real data collected from register of patients admitted to Başkent 

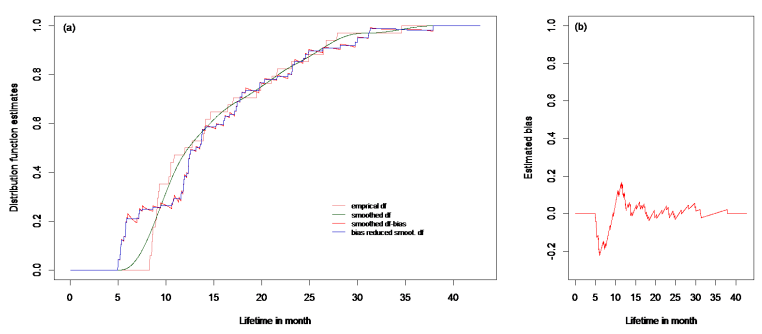

Fig. 2. Distribution function estimates.
University Hospital between January 1, 1990 and November 30, 1992 [11].

In Fig. 2 , we see $F_{n}(x), \tilde{F}_{n, h}(x), \tilde{F}_{n, h}(x)-$ bias and $\tilde{\tilde{F}}_{n, h}(x)$ all together. Since we subtract the estimated bias from the smoothed distribution function, we obtained a new function which does not satisfy distribution function property. By using interpolation we deduce the distribution function property again. We see that situation in the graph obviously.

TABLE II

Sampling distribution of estimated bias for some quantile values, $\operatorname{Weibull}(1.5,1)$, $n=40$, repetition number $=1000$.

\begin{tabular}{|c|c|c|c|c|c|c|}
\hline Contamination & & $\tilde{\tilde{Q}}_{0.125}$ & $\tilde{\tilde{Q}}_{0.25}$ & $\tilde{\tilde{Q}}_{0.50}$ & $\tilde{\tilde{Q}}_{0.75}$ & $\tilde{\tilde{Q}}_{0.875}$ \\
\hline \multirow{2}{*}{ no } & $\begin{array}{c}\text { Mean of } \\
\text { estimated bias }\end{array}$ & -0.039962 & 0.004654 & 0.025358 & 0.026310 & 0.022103 \\
\hline & $\begin{array}{c}\text { Variance of } \\
\text { estimated bias }\end{array}$ & 0.000129 & 0.001464 & 0.001569 & 0.000768 & 0.000507 \\
\hline \multirow{2}{*}{$5 \%$} & $\begin{array}{c}\text { Mean of } \\
\text { estimated bias }\end{array}$ & -0.047434 & 0.002409 & 0.028092 & 0.028447 & 0.020214 \\
\hline & $\begin{array}{c}\text { Variance of } \\
\text { estimated bias }\end{array}$ & 0.001135 & 0.001853 & 0.001599 & 0.000780 & 0.000450 \\
\hline \multirow{2}{*}{$10 \%$} & $\begin{array}{c}\text { Mean of } \\
\text { estimated bias }\end{array}$ & -0.053744 & -0.000895 & 0.034993 & 0.028325 & 0.007458 \\
\hline & $\begin{array}{c}\text { Variance of } \\
\text { estimated bias }\end{array}$ & 0.001080 & 0.002081 & 0.001552 & 0.000606 & 0.000680 \\
\hline
\end{tabular}

TABLE III

The ratio of variances of estimated bias of quantile estimators and median.

\begin{tabular}{c|c|c|c|c|c|c|c|c}
\hline \hline & \multicolumn{4}{|c|}{ Weibull $(1.5,1)$} & \multicolumn{4}{c}{ Weibull $(5,1)$} \\
\hline Contamin. & $\frac{V\left(\tilde{\tilde{Q}}_{0.125}\right)}{V\left(\tilde{\tilde{Q}}_{0.50}\right)}$ & $\frac{V\left(\tilde{\tilde{Q}}_{0.25}\right)}{V\left(\tilde{\tilde{Q}}_{0.50}\right)}$ & $\frac{V\left(\tilde{\tilde{Q}}_{0.75}\right)}{V\left(\tilde{\tilde{Q}}_{0.50}\right)}$ & $\frac{V\left(\tilde{\tilde{Q}}_{0.875}\right)}{V\left(\tilde{\tilde{Q}}_{0.50}\right)}$ & $\frac{V\left(\tilde{\tilde{Q}}_{0.125}\right)}{V\left(\tilde{\tilde{Q}}_{0.50}\right)}$ & $\frac{V\left(\tilde{Q}_{0.25}\right)}{V\left(\tilde{\tilde{Q}}_{0.50}\right)}$ & $\frac{V\left(\tilde{Q}_{0.75}\right)}{V\left(\tilde{\tilde{Q}}_{0.50}\right)}$ & $\frac{V\left(\tilde{Q}_{0.875}\right)}{V\left(\tilde{\tilde{Q}}_{0.50}\right)}$ \\
\hline $0 \%$ & 0.082 & 0.933 & 0.489 & 0.323 & 3.577 & 0.622 & 0.724 & 0.490 \\
$5 \%$ & 0.710 & 1.140 & 0.487 & 0.281 & 0.440 & 0.625 & 0.729 & 0.494 \\
$10 \%$ & 0.696 & 1.367 & 0.390 & 0.438 & 0.422 & 0.649 & 0.565 & 0.408
\end{tabular}

Estimated bias for low quantile values is very variable as it is seen from Table V and Fig. 2. So it has strong effect on the estimate based on smoothed bias reduced distribution function.

\section{Conclusions}

In this study, bias reduced quantile-based estimators are obtained for Weibull distribution with varied parameters for different contamination level. Based on a simulation study, empirical, smoothed and bias reduced distribution functions are achieved and their graphs are drawn. Sampling distribution of roughness estimator and of bandwidth estimator is obtained and their graphs are given. The sampling distribution of roughness is strongly right skewed for all contamination level and for considered two different Weibull distributions. The sampling distribution of bandwidth is slightly right skewed for all contamination level and for considered two different Weibull distributions. In simulation study, we obtained compatible results with a structure of distribution.

The sampling distribution of estimated bias related to quantile estimator of order $0.125 Q(0.125)$ is right skewed in the case of $\operatorname{Weibul}(1.5,1)$ for all contamination levels. On the other hand, the sampling distribution of estimated bias related to other considered quantile estimators of any order is almost symmetric in the cases $\operatorname{Weibul}(1.5,1)$ and Weibul$(5,1)$ for all contamination levels.

Bias reducing approach seems to be very useful, it can be applied easily. We thought that there is need to make new study directed to determine for which estimators the bias reducing approach is effective. Furthermore the programs for simulation studies and application are coded in software $\mathrm{R}$ without using any robust packages. 
TABLE IV

Sampling distribution of some quantile-based estimators with $n=40$, repetition number $=1000$.

\begin{tabular}{c|c|c|c|c|c|c|c|c}
\hline \hline & \multicolumn{9}{|c|}{ Weibull $(1.5,1)$} & \multicolumn{2}{c}{ Weibull $(5,1)$} \\
\cline { 2 - 8 } & $0 \%$ contamination & \multicolumn{2}{|c}{$5 \%$ contamination } & \multicolumn{1}{c}{ 10\% contamination } & \multicolumn{2}{c}{$5 \%$ contamination } \\
\cline { 2 - 8 } & Mean & Variance & Mean & Variance & Mean & Variance & Mean & Variance \\
\hline$\tilde{\tilde{Q}}_{0.125}$ & 0.186493 & 0.011263 & 0.180123 & 0.014471 & 0.158992 & 0.016854 & 0.676510 & 0.003639 \\
$\tilde{\tilde{Q}}_{0.25}$ & 0.449009 & 0.006608 & 0.480423 & 0.008124 & 0.497392 & 0.009245 & 0.798260 & 0.002464 \\
$\tilde{\tilde{Q}}_{0.50}$ & 0.823543 & 0.011203 & 0.889423 & 0.014378 & 0.956442 & 0.018891 & 0.967110 & 0.001920 \\
$\tilde{\tilde{Q}}_{0.75}$ & 1.292809 & 0.022930 & 1.432673 & 0.040414 & 1.610792 & 0.076405 & 1.138560 & 0.002846 \\
$\tilde{\tilde{Q}}_{0.875}$ & 1.698059 & 0.041995 & 2.000773 & 0.108944 & 2.341142 & 0.135284 & 1.350010 & 0.118629 \\
$I \tilde{\tilde{Q}} R$ & 0.843800 & 0.019222 & 0.952250 & 0.034277 & 1.113400 & 0.068420 & 0.340300 & 0.003841 \\
$\tilde{\tilde{O}}_{n}$ & 0.110660 & 0.000000 & 0.138456 & 0.000000 & 0.168254 & 0.000000 & 0.005274 & 0.000000 \\
$\tilde{\tilde{Q}} S_{n}$ & 0.153840 & 0.011543 & 0.211437 & 0.014052 & 0.262438 & 0.013712 & 0.053630 & 0.040984
\end{tabular}

TABLE V

Empirical, smoothed and smoothed bias reduced quantile-based estimates for the lifetime data of kidney transplantation.

\begin{tabular}{c|c|c|c|c|c|c|c|c}
\hline \hline & $Q_{n}(0.125)$ & $Q_{n}(0.25)$ & $Q_{n}(0.50)$ & $Q_{n}(0.75)$ & $Q_{n}(0.875)$ & $I Q R$ & $Q S_{n}$ & $O S_{n}$ \\
\hline Emprical & 0.5465 & 1.0465 & 3.9965 & 11.9299 & 16.8965 & 10.8833 & 0.4579 & 0.5780 \\
Smoothed & 0.4652 & 1.0799 & 4.3299 & 11.8965 & 16.9799 & 10.8167 & 0.3991 & 0.4941 \\
S.bias-red & -2.4201 & 0.6132 & 5.1299 & 11.7799 & 16.5465 & 11.1667 & 0.1910 & 0.2039 \\
Estimated bias & -0.1237 & -0.0319 & 0.0855 & -0.0174 & -0.0273 & - & - & -
\end{tabular}

\section{References}

[1] E.A. Nadaraya, Theor. Probab. Appl. 9, 497 (1964).

[2] A. Azzalini, Biometrika 68, 326 (1981).

[3] L.T. Fernholz, J. Stat. Plan. Infer. 57, 29 (1997).

[4] M. Hubert, I. Gijbels, D. Vanpaemel, Test 22, 448 (2013).

[5] N. Gündüz, E. Başar, C. Aydın, Acta Phys. Pol. A 128, B-203 (2015).

[6] G. Brys, M. Hubert, A. Struyf, J. Comput. Graph Stat. 13, 996 (2004).

[7] G. Brys, M. Hubert, A. Struyf, in: Developments in robust statistics, Vol. 114, Eds. R. Dutter, P. Filzmoser, U. Gather, P.J. Rousseeuw, Physika Verlag, Heidelberg 2003, p. 98.
[8] V. Epanechnikov, Theor. Probab. Appl. 14, 153 (1969).

[9] M.P. Wand, M.C. Jones, Kernel Smoothing, Chapman \& Hall/CRC Monographs on Statistics \& Applied Probability, 1994.

[10] B.W. Silverman, Density estimation for statistics and data analysis, Chapman and Hall, London 1986.

[11] E. Başar, Unpublished Ph.D. Thesis, Sci. Inst. of Hacettepe of Uni., Ankara, Turkey 1993. 